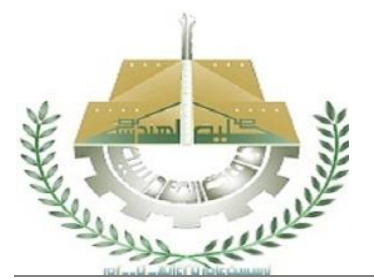

\title{
NUMERICAL INVESTIGATION OF MIXED CONVECTION BY NON-CONCENTRIC POSITIONS OF ROTATING CIRCULAR CYLINDER IN SQUARE ENCLOUSRE
}

\author{
Farooq Hassan Ali, \\ Mechanical Engineering Department, College of Engineering, University of Babylon 520001, Hilla, Iraq. \\ Email:farooq_hassan77@yahoo.com
}

Duraid F. Maki, Mechanical Engineering Department, College of Engineering, University of Babylon 520001, Hilla, Iraq. E mail:duraid.maki@yahoo.com

\begin{abstract}
Mushtaq Fasiel
Mechanical Engineering Department, College of Engineering, University of Al- Qadisiyah, Diwanyia- Iraq. E mail: mushtaq.fisaal@qu.edu.iq
\end{abstract}

Received on 4 January 2018 Accepted on 24 January 2018 Published on 14 May 2018

DOI: 10.30772/qjes.v11i1.521

\begin{abstract}
In this work numerical investigation of mixed convection by non-concentric different positions of rotating circular cylinder in square enclosure is investigated. The assumption of using six rotating cylinders at constant temperature $T_{h}$ and square closed enclosure with temperature $T_{c}$ for all walls are maintained. Continuity, Momentum and energy governing equations are solved by using finite volume technique using AnsysFluent 16 commercial code with two dimension and steady state case. Richardson number $\left(R i=G r / R e^{2}\right)$ varying over the wide range of $0.1,1,10,100,1000$ and $\infty$. This research investigate the effect of changing the rotating circular cylinder positions along the vertical centerline on the fluid flow and heat transfer inside the enclosure. Six different positions are tested. The phenomenon inside the square enclosure is mathematically analyzed. A streamlines, isothermal patterns, local Nusselt numbers, and average Nusselt numbers are calculated. The analytical results give that, the Richardson numbers is playing the dominant role on temperature distributions and flow patterns inside the enclosure. The average Nusselt number variation demonstrates the best location of rotating cylinder which gives best heat transfer value. The bottom left corner $\left(P_{4}\right)$ is the best location for hot rotating cylinders. While the rotating in middle right $\left(P_{6}\right)$ is the inferior position.
\end{abstract}

Keywords: Mixed Convection, Square Cavity, Rotating Circular Cylinder, Richardson Number, Ansys-Fluent. 


\section{AL-QADISIYAH JOURNAL FOR ENGINEERING SCIENCES}

\section{INTRODUCTION}

Since one century the phenomenon of mixed heat transfer inside closed enclosure is studied experimentally and numerically. Due to appear of this phenomenon in many thermal engineering applications, it was and still the focus of research and study.

Although one century ago, the study of heat convection from rotating cylinders inside closed enclosure is promising and continued. This heat convection with variable temperature for rotating cylinders and outside surfaces temperatures has widely applications in internal combustion, clean solar energy, air conditioning, boilers, etc. The reviewing of critical literatures for past decade shows that most of modern critical researches focused on numerical mathematical techniques to solve the set of governing equations using the high development in programming, used different shapes of enclosure, and cylinders characteristics. The steady and 2- D numerical solution by different techniques are widely used to solve the natural and mixed heat convection from cylinders set inside close enclosure [1-11,13].

The unsteady investigation by numerical solution technique is studied also for this phenomenon [12]. The laminar, steady, mixed convection flow, and two dimension is carried out experimentally [13]. Different enclosure shapes are investigated, the enclosure of square shape [1,3-6, 8-11], trapezoidal shape [2], and triangle shape $[4,7,13]$ are widely studied. All literatures showed that different radius cylinders in different arranging are subjected to convection heat transfer with the media which fill the space between cylinders and close enclosure [1-13].

The numerical or experimental study of mixed heat transfer problem for cylinders arranged in symmetric system parameters has different parameters. The Rayleigh number, Prandtl number, Nusselt number, Grashof number, Richardson number, Hartmann number, conductivity ratio, working fluid, Reynolds number, and convection are studied [1-13].

Different fluid working media are subjected between the cylinder and boundary of close enclosure [1-4, 811]. Due to prominence of nano fluid application researches, studies on nano fluids are carried out in this field $[5,7,13]$.

In present work, mixed convection heat transfer by non-concentric positions of rotating circular cylinder in square enclosure are carried out. The study goals to find optimum position of peak heat transfer.

\section{MODELING AND SYSTEM LAYOUT}

The system description in the present work is shown in Fig.1. The system consists of a square enclosure with sides of length $\mathrm{H}$, the rotating cylinders have radius $(\mathrm{R}=0.2 \mathrm{H})$ is located in six different positions and rotating. The outer walls of the square enclosure was kept at cold temperature of $T_{c}$, whereas the interior cylinder wall was kept at hot temperature $\mathrm{Th}_{\mathrm{h}}$. Under the influence of interaction between the gravity, the enclosure cold walls temperature and hot temperature at the surface of rotating circular cylinder at different positions leads to a mixed convection problem. Due to the nonslipup boundary condition for velocity on it is surface and the rotating cylinder, induced a forced flow, the overall resulting situation being a mixed convection problem. The region between the circular rotating cylinder and enclosure walls is filled by air. The air density is changed due to temperatures variation only. Boussinesq approximation are used for density on the buoyancy term for temperature dependent. Only the remaining thermo physical properties of the fluid are assumed to be constant and listed in the table (1). The flow is assumed to be laminar. No thermal radiation heat transfer between the walls and the surface of circular cylinder, and the fluid assumed to be radioactively non contributing. Air is taken as a working fluid with Prandtl number (0.71) and the reference temperature is taken as $300 \mathrm{~K}\left(T_{r}=\frac{T_{0}+T_{i}}{2}\right)$. The energy terms due to viscous dissipation and change of temperature due to reversible deformation(work of pressure forces) are not taken into account in the present study. 


\begin{tabular}{|c|c|}
\hline \multicolumn{2}{|c|}{ Table (1) Properties of Air } \\
\hline Variable & Value (Unit) \\
\hline$\rho$ & $1.1614 \mathrm{~kg} / \mathrm{m}^{3}$ \\
\hline $\mathrm{C}_{\mathrm{p}}$ & $1007 \mathrm{~J} / \mathrm{kg} . \mathrm{K}$ \\
\hline$\mu$ & $1.846^{*} 10^{-5} \mathrm{~N} . \mathrm{s} / \mathrm{m}^{2}$ \\
\hline$v$ & $1.589^{*} 10^{-5} \mathrm{~m}^{2} / \mathrm{s}$ \\
\hline $\mathrm{k}$ & 0.026182 \\
\hline$\alpha$ & $2.25^{*} 10^{-5} \mathrm{~m}^{2} / \mathrm{s}$ \\
\hline
\end{tabular}

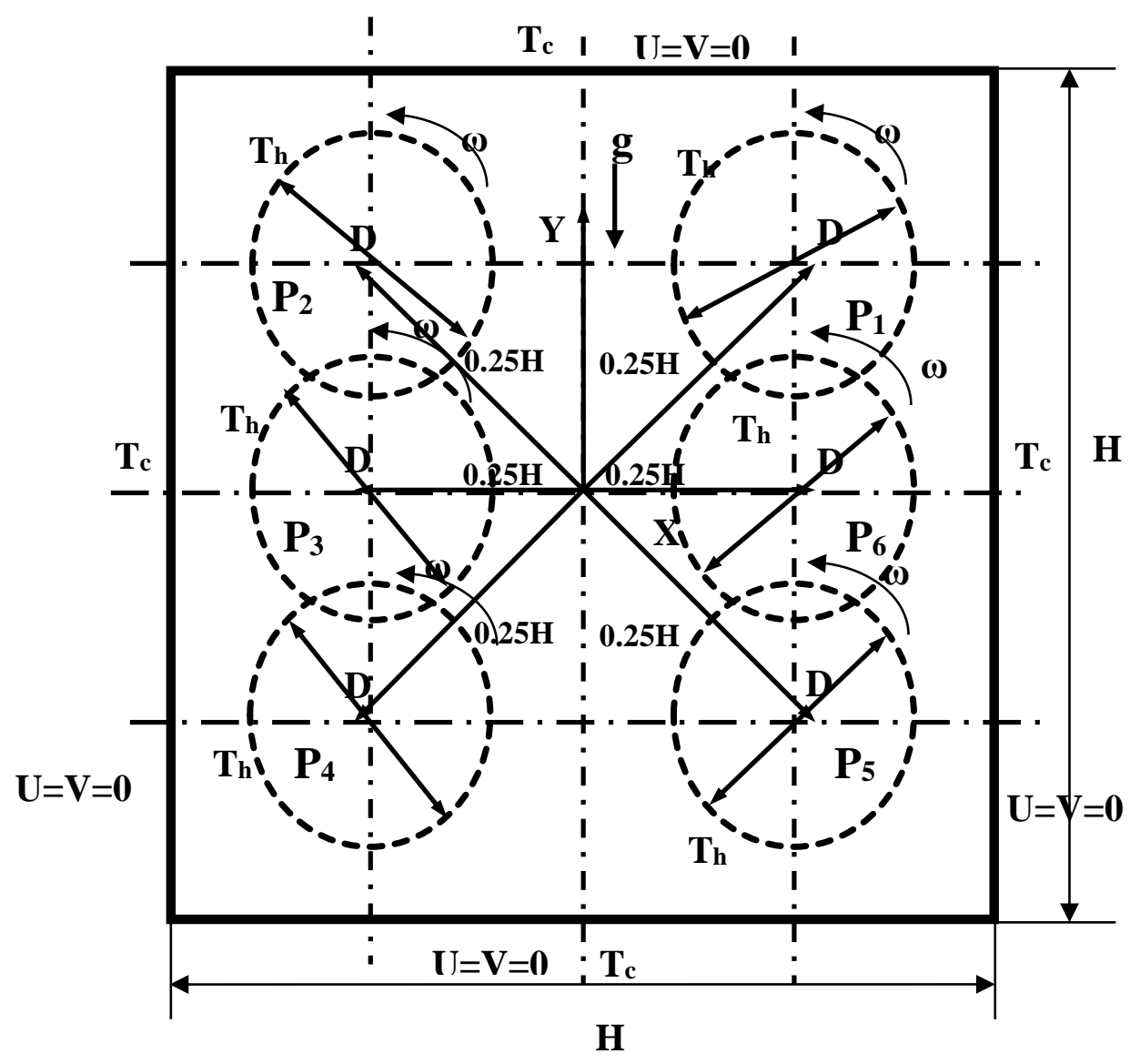

Fig. 1. Geometry description of the module 


\section{NUMERICAL MODELING EQUATION}

\section{Governing Equations:}

In the study Ansys Fluent package was used to solve the governing equations. Control volume techniques was used to transfer the governing equation to algebraic equations. The numerical solutions based on control volume approach where the integration of the governing equation in each control volume, and then discretization of the equations which transfer any quantity to control volume[15]. The governing equation continuity, momentum and energy equations for laminar, incompressible, two dimensional and steady state can written as follows:

\section{Continuity Equation :}

$\frac{\partial}{\partial x}(\rho u)+\frac{\partial}{\partial y}(\rho v)=0$

Momentum Equations in $\mathbf{x}, \mathbf{y}$ directions :

$$
\begin{aligned}
& \rho\left[u \frac{\partial u}{\partial x}+v \frac{\partial u}{\partial y}\right]=-\frac{\partial P}{\partial x}+\mu\left(\frac{\partial^{2} u}{\partial x^{2}}+\frac{\partial^{2} u}{\partial x^{2}}\right) \\
& \rho\left[u \frac{\partial v}{\partial x}+v \frac{\partial v}{\partial y}\right]=-\frac{\partial p}{\partial y}+\rho g+\mu\left(\frac{\partial^{2} v}{\partial x^{2}}+\frac{\partial^{2} v}{\partial y^{2}}\right)
\end{aligned}
$$

\section{Energy Equation:}

$u \frac{\partial T}{\partial x}+v \frac{\partial T}{\partial y}=\alpha\left(\frac{\partial^{2} T}{\partial x^{2}}+\frac{\partial^{2} T}{\partial y^{2}}\right)$

The above equations when transform to the Finite Volume Form becomes

Continuity equation

$\rho \nabla \cdot \vec{V}=0$

Momentum equation

$\frac{\partial\left(\rho u^{2}\right)}{\partial x}+\frac{\partial(\rho v u)}{\partial y}=-\frac{\partial p}{\partial x}+\frac{\partial}{\partial x}\left(\lambda \nabla \cdot \vec{V}+2 \mu \frac{\partial u}{\partial x}\right)+\left[\frac{\partial}{\partial y}\left(\mu\left(\frac{\partial v}{\partial x}+\frac{\partial u}{\partial y}\right)\right]+\rho f_{x}\right.$
$\frac{\partial(\rho u v)}{\partial x}+\frac{\partial\left(\rho v^{2}\right)}{\partial y}=-\frac{\partial p}{\partial y}+\frac{\partial}{\partial x}\left[\mu\left(\frac{\partial v}{\partial x}+\frac{\partial u}{\partial y}\right)\right]+\frac{\partial}{\partial y}\left(\lambda \nabla \cdot \vec{V}+2 \mu \frac{\partial v}{\partial y}\right)+\rho f_{y}$

The energy equation

$\frac{\partial}{\partial x}\left(k \frac{\partial T}{\partial x}\right)+\frac{\partial}{\partial y}\left(k \frac{\partial T}{\partial y}\right)=\frac{\partial(u p)}{\partial x}+\frac{\partial(v p)}{\partial y}-\frac{\partial\left(u \tau_{x x}\right)}{\partial x}-\frac{\partial\left(u \tau_{y x}\right)}{\partial y}-\frac{\partial\left(v \tau_{x y}\right)}{\partial x}-\frac{\partial\left(v \tau_{y y}\right.}{\partial y}-\rho \vec{f} \cdot \vec{V}$

Where $\mu$ is the molcular viscosity coefficient and $\lambda$ is the bulk viscosity coefficient. Stokes made the hypothesis that $\lambda=2 / 3 \mu$.

The boundary conditions are assumed to be:

1- $\mathrm{u}=\mathrm{v}=0$ on the outer square enclosure walls

2- $u=\omega r \cos \theta, v=\omega r \sin \theta$ on the inner rotating cylinder 
$\mathrm{T}_{i}=325 \mathrm{~K}$ on the inner cylinder (hot wall), $\mathrm{T}_{0}=275 \mathrm{~K}$ on outer cylinder (cold wall). The dimensionless parameter appearing in the forgoing equations are the space coordinates, $R i=\frac{G r}{\operatorname{Re}^{2}}$, where $\mathrm{Re}$ is the Reynolds number $\left(\operatorname{Re}=\frac{(\omega R) D}{v}\right)$, where $\mathbf{D}$ is the diameter of the cylinder. Gr $=\frac{\mathrm{g} \beta \Delta \mathrm{T} \rho^{2} \mathrm{H}^{3}}{\mu^{2}}$

\section{Heat Transfer Calculations}

The local Nusselt number and average Nusselt number are obtained on the surface of the heated rotating cylinder from temperature gradient by the following equations.

$$
\begin{aligned}
& N u=\left.\frac{\partial T}{\partial r}\right|_{R} \\
& \overline{N u}=\frac{1}{2 \pi} \int_{0}^{2 \pi} N u_{r}
\end{aligned}
$$

\section{METHOD OF SOLUTION}

In this work the continuity, momentum and energy equations are periodicity solved by control volume techniques built in computational fluid dynamic (CFD) by using commercial code (ANSYS-FLUENT 16). The model geometry preprocessing by using Workbench program, then divided into mapped finite volume using mesh generation in the same program (Workbench program). Fig.2 describe the mapped mesh of the present study, when rotating cylinder in position four. The implicit pattern with second-order precision to control the solution strategies have choosen. To discretizing the continuity, momentum and energy equations, Presto method is employed for the pressure equation, while the Quick method is employed for both momentum and energy equations. Moreover, the pressure-velocity coupling is employed depends on the simple algorithms. The convergence criterion are to be chosen is $10^{-6}$ for continuity and momentums equations but for energy equation is taken $10^{-8}$.

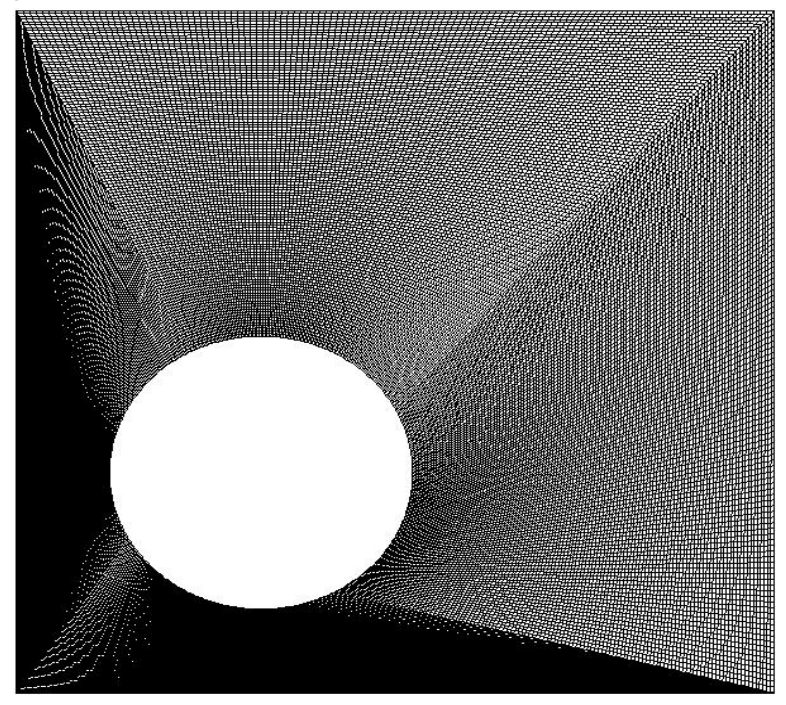

Figure 2: Mesh Generation 


\section{GRID INDEPENDENCE AND CODE VALIDATION}

Grid verification is described in Fig.3, the stability of the examined work is achieved at 56459 elements of finite control volume with $\left(140^{*} 140\right)$ division where the deviations in average Nusselt number reaches to $1.67^{*} 10^{-3}$. To ensure the results of the commercial code, validations was established with Ref(14). Fig.4 gives the results of this validation, good validation was achieved in stream, isothermal and heat transfer. The mesh is mapped with structured elements.

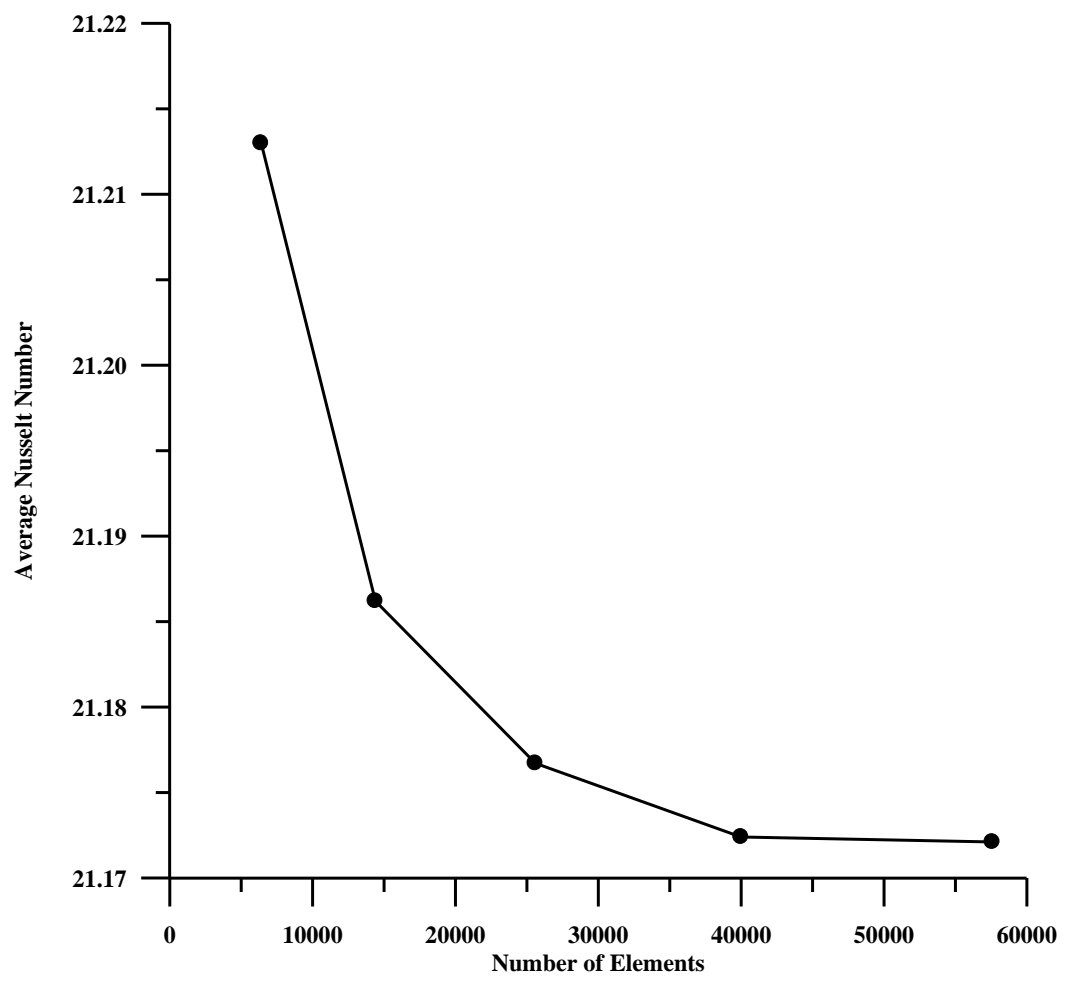

Figure : Verification of mesh generation

\section{RESULTS AND DISCUSSION}

The aim of numerical solution for mathematical governing equations is find the best position (best heat convection) for the cylinder inside the enclosure where six different positions are tested. Air is the working fluid between hot cylinder and square enclosure surfaces. Mix of natural heat convection and forced heat convection is investigated where Richardson number is varied at $0.1,1,10,100,1000$, and $\infty$. The variation is studied between the maximum forced convection heat transfer at $\mathrm{Ri}=0.1$ and max. natural convection at $\mathrm{Ri}=\infty$. The mathematical equation of $\mathrm{Ri}=\frac{G r}{R e^{2}} \quad$ given above.

Hence Numerator is representing the free heat convection while the denominator gives the forced heat convection due to effect of hot cylinder rotating speed. Figures from 5 to 10 show the effect of hot cylinder position on the stream line and isothermal lines pattern respectively.

Fig (5) shows streamlines for different Richardson Number at Position Number One. The high effect of hot cylinder speed $(\omega)$ is appeared. It can be observed that small vortex lines due to collision between the stream lines and right walls where the hot rotating cylinder effect on the 


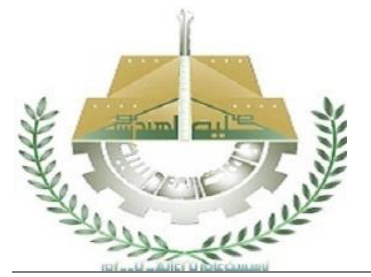

\section{AL-QADISIYAH JOURNAL FOR ENGINEERING SCIENCES}

Vol. 11 , No. 1

ISSN: $1998-4456$

stream lines and didn't give the vortex enough chance to grow. High $\omega$ means high value of $(\mathrm{Re})$. High value of $\mathrm{Re}$ indicates high effect of force convection and low effect to the natural convection. This meaning is clear appearing in isothermal lines distribution as give in fig (5). The shape of isothermal lines shows line distribution in working fluid area.

\section{Stream lines Contour}
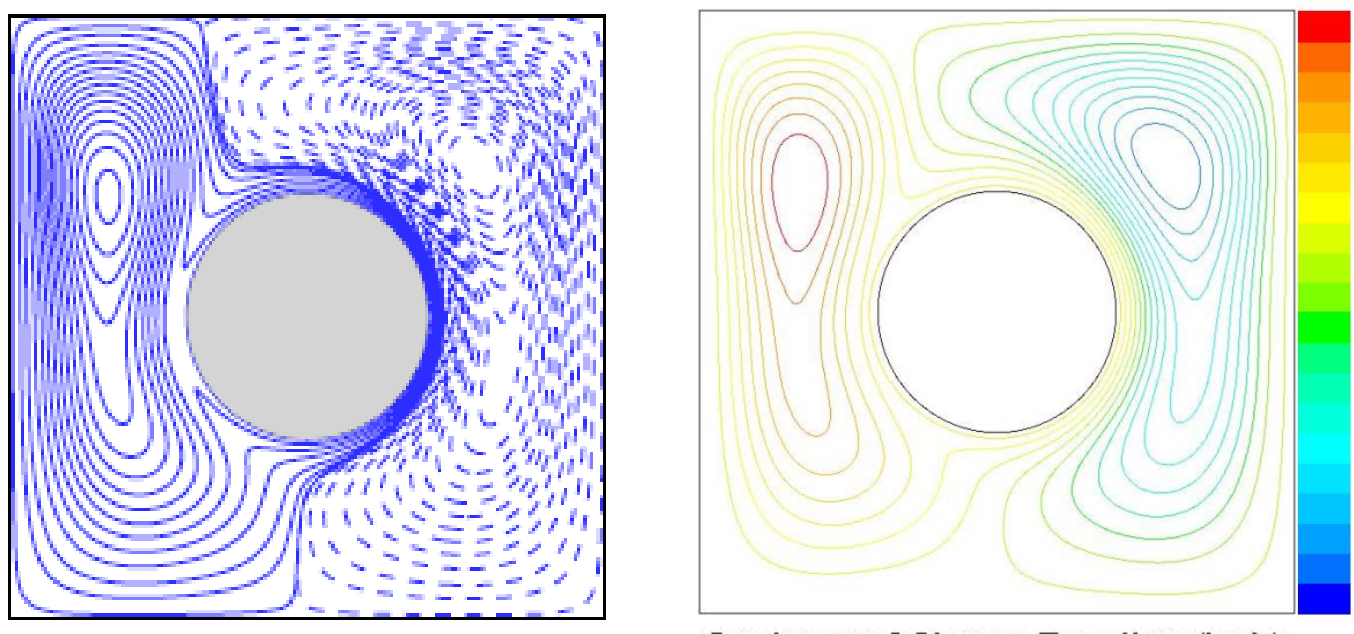

$4.13 \mathrm{e}-04$

$3.71 \mathrm{e}-04$

$3.51 \mathrm{e}-04$

$3.30 \mathrm{e}-04$

3.10e-04

2.89e-04

$2.68 \mathrm{e}-04$

$2.48 \mathrm{e}-04$

$2.27 \mathrm{e}-04$

$2.06 \mathrm{e}-04$

$1.86 \mathrm{e}-04$

$1.65 \mathrm{e}-04$

$1.44 \mathrm{e}-04$

$1.24 \mathrm{e}-04$

$1.03 \mathrm{e}-04$

8. $25 \mathrm{e}-05$

6. $19 \mathrm{e}-05$

4. $13 \mathrm{e}-05$

Contours of Stream Function $(\mathrm{kg} / \mathrm{s})$

\section{Isothermal Contour}
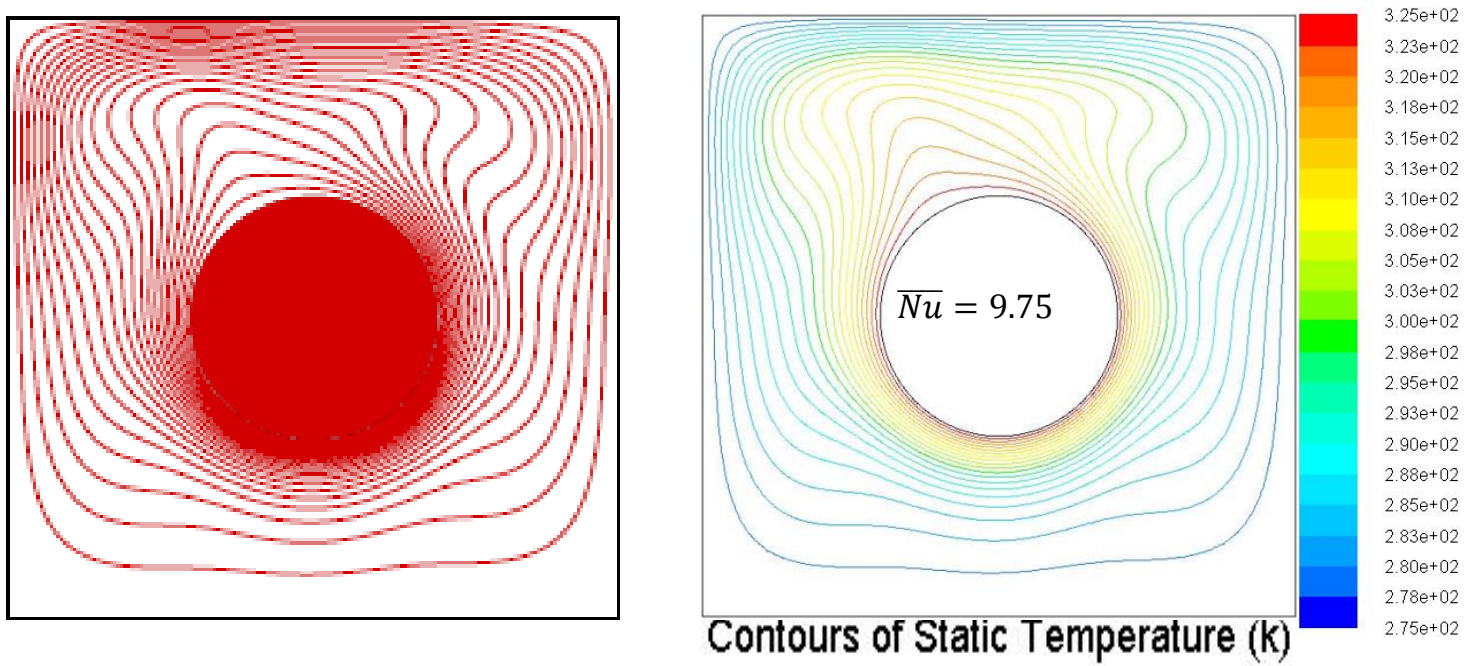

Figure 4: Comparison of streamline, isothermal line and heat transfer average Nusselt number $(\overline{N u})$ for present study and $\mathrm{ref}(14)$, For $\mathrm{H} / \mathrm{D}=2.5, \mathrm{R} a=10^{5}, \mathrm{R} i=100$ 


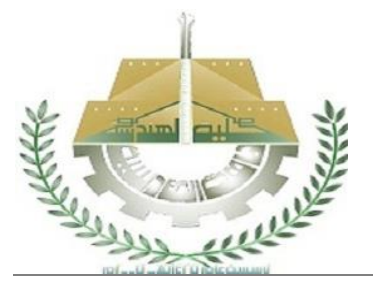

\section{AL-QADISIYAH JOURNAL FOR ENGINEERING SCIENCES}

Vol. 11, No. 1

ISSN: $1998-4456$
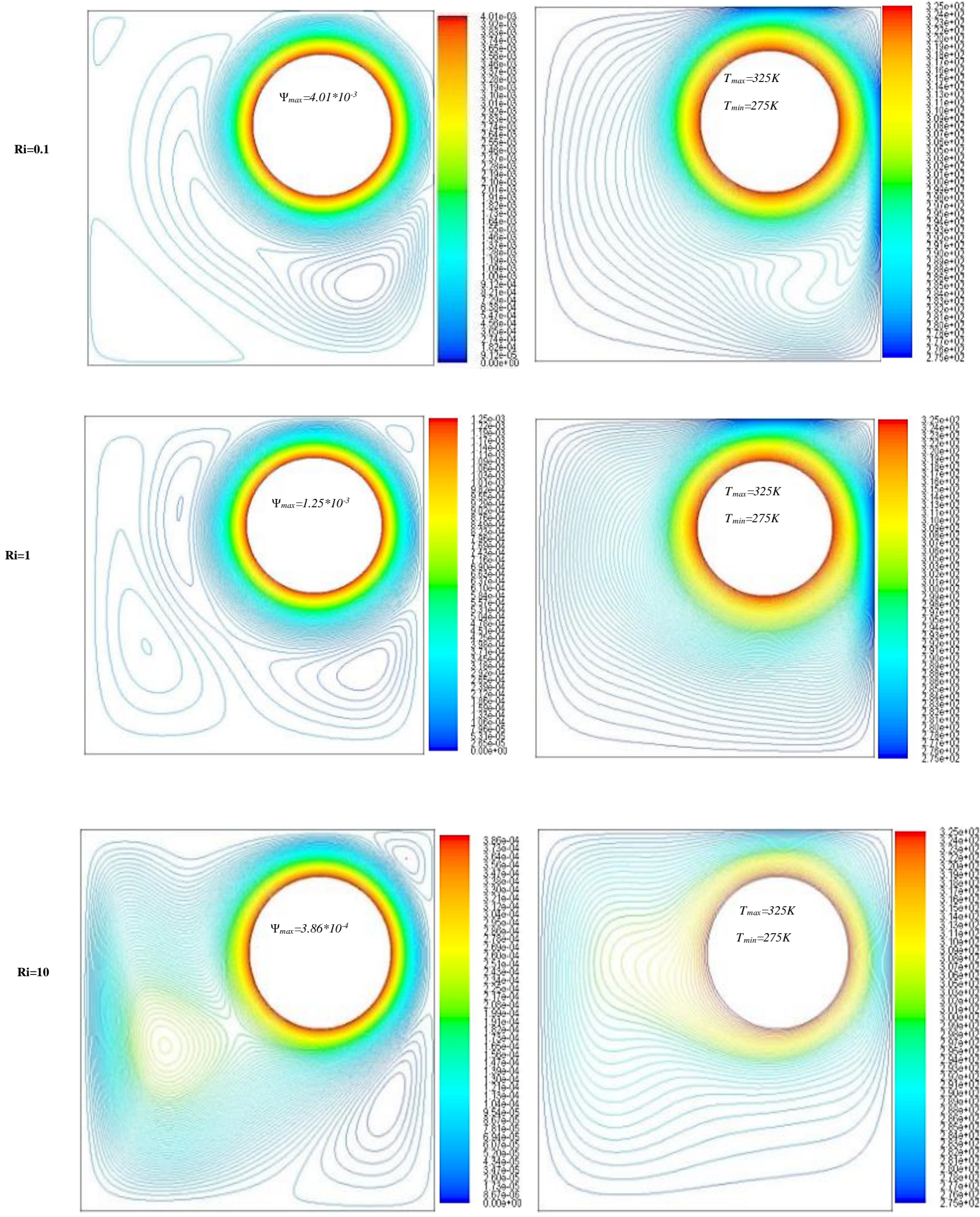


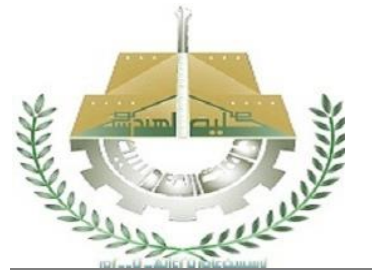

\section{AL-QADISIYAH JOURNAL FOR ENGINEERING SCIENCES}
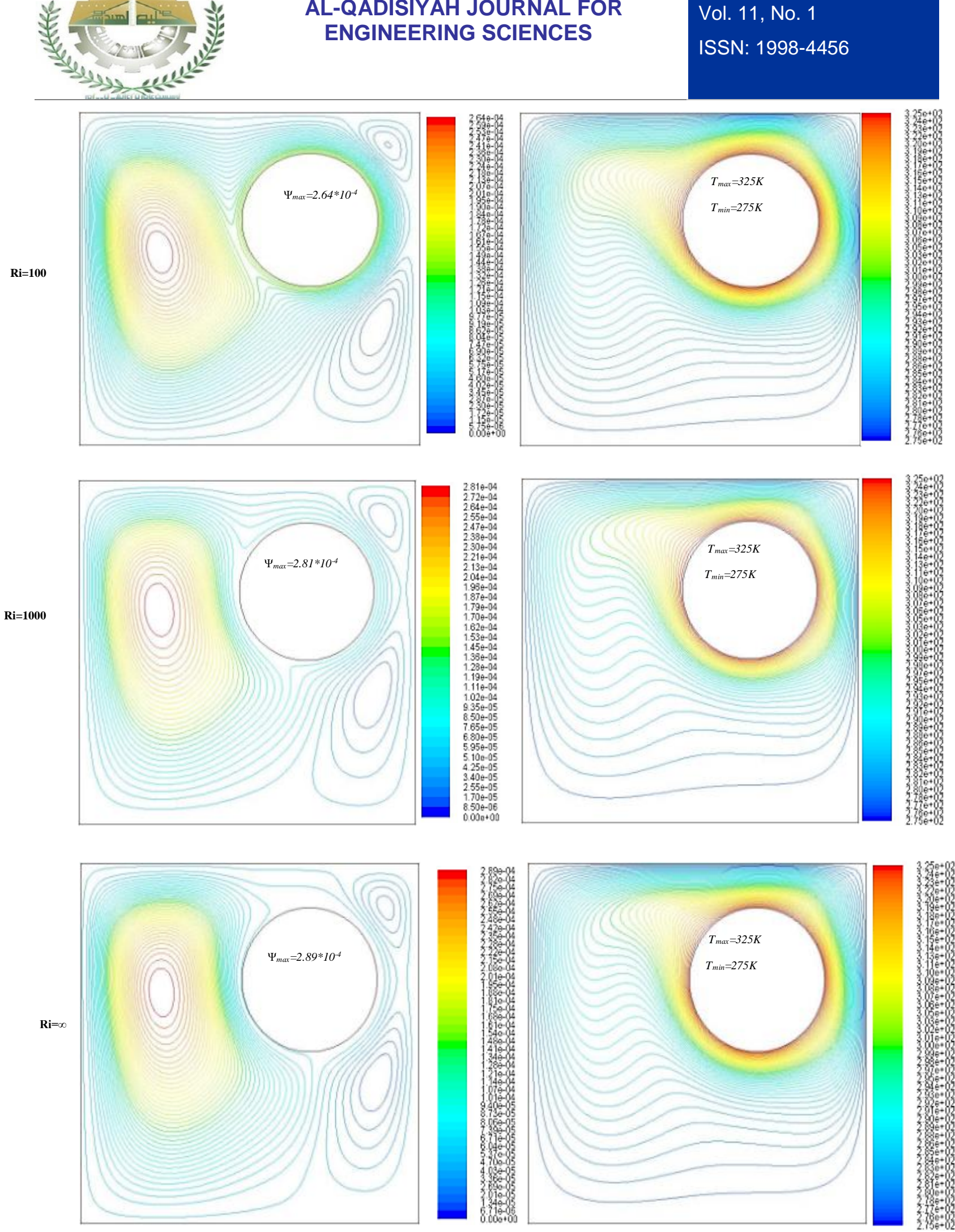

Fig. 5. streamlines (on the left) and Isotherms (on the right) for different Richardson Number at Position Number One 


\section{AL-QADISIYAH JOURNAL FOR ENGINEERING SCIENCES}

Fig (6) gives streamlines for different Richardson Number at Position Number two. The position of cylinder in upper left corner of square enclosure made tight area in the above of hot cylinder means the space is available under the cylinder and on right of it. At $\mathrm{Ri}=0.1$ ( $\mathrm{Ri}$ not equal zero because that means no difference in temperature between cylinder and enclaves walls on respective). The high anti clock wise rotation of hot cylinder is the mainly effect factor on convection heat transfer. Effect of heat transfer by natural convection is so weak. With the increasing in value of Ri which is meaning retarding in effect of rotating hot cylinder heat convection, the dense of arranged stream lines becomes quiet clear when comparison is held between streamlines distribution at $R \mathrm{i}=0.1$ and streamlines distribution at $R \mathrm{i}=1000$ and $\infty$. The yellow line and sky blue line in fig 6 show the effect of convection heat transfer on stream lines. At $\mathrm{Ri}-0.1$ and $\mathrm{Ri}=1$, these two lines are totally have cyclic shape around the hot cylinder which gives clear view about effect of force convection. At $\mathrm{Ri}=100,1000$, and $\infty$ these two lines moved gradually to separate from cylinder body from down points and move forward the space on right to form natural convection stream lines. At $\mathrm{Ri}=\infty$, it can be clearly observed a limited forced convection and wide dominant effect for natural convection. In isothermal lines, same scenario is reflected and merely noticeable the change of cylinder angular rotation speed effect from high forced convection at $\mathrm{Ri}=0.1$ and so low effect at $\mathrm{Ri}=\infty$ where the natural convection is appeared. Three nodes ending the initiation of natural conviction isothermal lines on upright, up left, and lift down of cylinder as shown in fig (6) and $\mathrm{Ri}=\infty$. These three nodes were started by only on node at $\mathrm{Ri}=100$ then two nodes at $\mathrm{Ri}=1000$ then at end i.e $\mathrm{Ri}=\infty$ by three nodes. This changeable in isothermal lines gave the full historic of heat convection transforming from forced convection to natural convection.

Fig (7) illustrates streamlines distribution (on the left) and Isotherms line distribution (on the right) for different Richardson Number at Position Number Three. The new position of hot cylinder gave it more space in above, down, and right side. Here, The shifting of heat convection from forced to natural is started early due to wide working fluid in around. So at $\mathrm{Ri}=0$ and $\mathrm{Ri}=1$, still the affection of forced convection is occurred. The shifting started at $\mathrm{Ri}=10$ where the stream lines started initiate new vortex due to natural heat convection and the influence of this convection grown. At $\mathrm{Ri}=\infty$, the dominant influence for natural convection only and no significant effect for rotating hot cylinder. From fig (7), isotherms lines show the effect transferring is already started at $\mathrm{Ri}=0.1$ marginally. This transfer in effect between forced and natural is happened gradually at $R i=1$ till $R i=\infty$. At $R i=\infty$, its observed that only natural convection is effected the heat transfer process and isotherm lines initiated distinguishing are in the right upper position where working fluid density is appear too.

In fig (8), provides the streamlines distribution shapes (on the left) and Isotherms lines (on the right) for different Richardson Number at Position Number Four. Position four mains at lift lower corner of square enclave see fig (1). In this position the working fluid has only space at top and right of hot cylinder in meanwhile other cylinder side so close from enclave walls where no space. Stream lines distribution showed effect of forced convection at $\mathrm{Ri}=0.1$ and 1 . The natural convection formed weak one vortex on the top of cylinder which indicates the weakness of natural heat convection. At $R \mathrm{i}=10$, the influence of natural convection is started increase and the stream lines became more dense around vortex till initiated second vortex on the lift of the first one and gradually decrease the influence of forced convection. At $\mathrm{Ri}=\infty$, slow rotation of hot cylinder gave quiet limited effect to forced convection whereas the natural heat convection at high rate due to that slow rotation and due to wide area availability in front the natural convection to effect easily and heavy air density at bottom push the hot and light air to the top. Moving of air due to its density difference is compatible with cylinder rotation direction which is helpful matter 


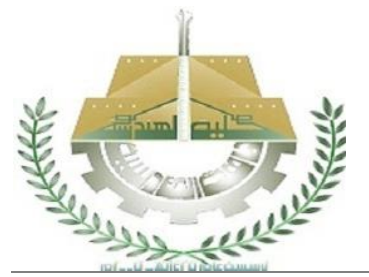

\section{AL-QADISIYAH JOURNAL FOR ENGINEERING SCIENCES}

Vol. 11, No. 1

ISSN: $1998-4456$
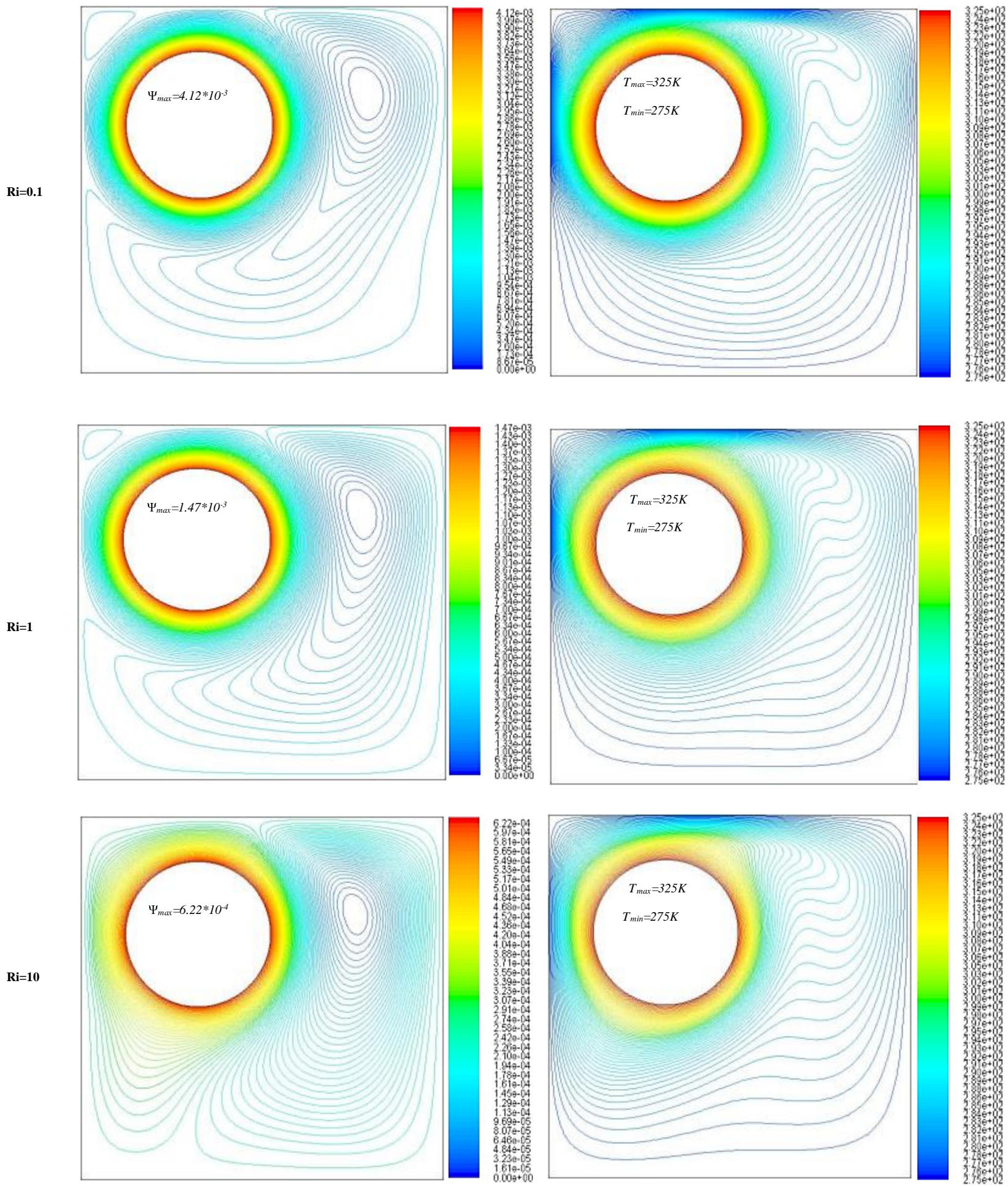


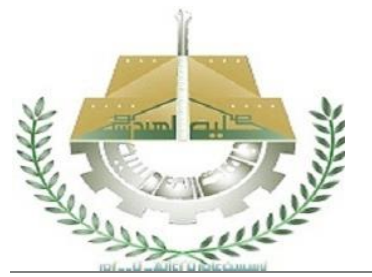

\section{AL-QADISIYAH JOURNAL FOR ENGINEERING SCIENCES}

Vol. 11, No. 1

ISSN: $1998-4456$
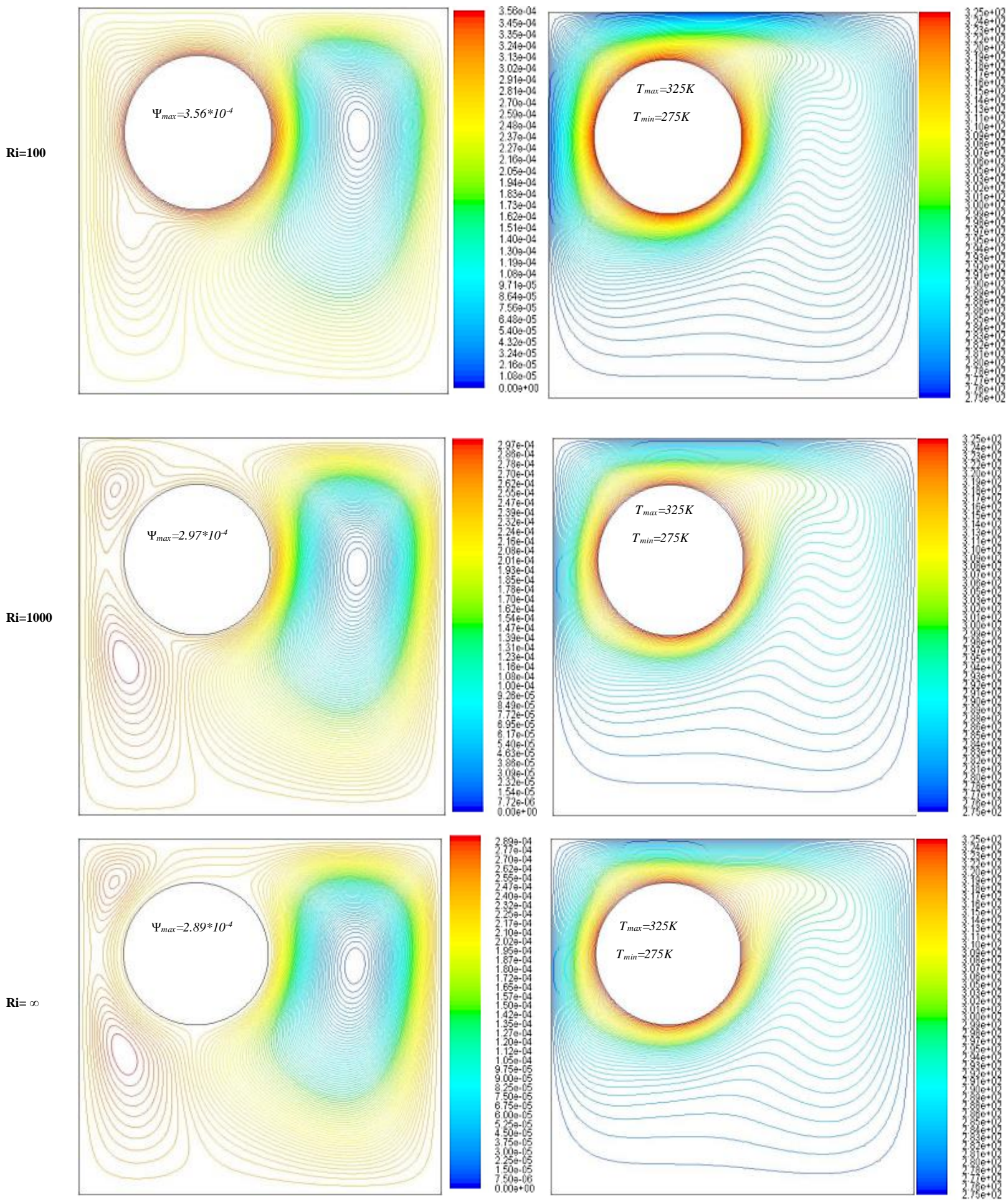

Figure 6: streamlines (on the left) and Isotherms (on the right) for different Richardson Number at Position Number Two 


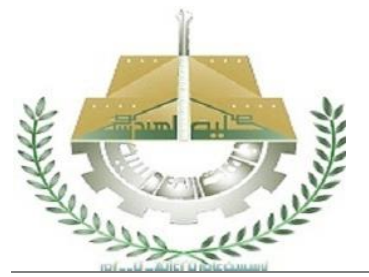

\section{AL-QADISIYAH JOURNAL FOR ENGINEERING SCIENCES}

Vol. 11, No. 1

ISSN: 1998-4456
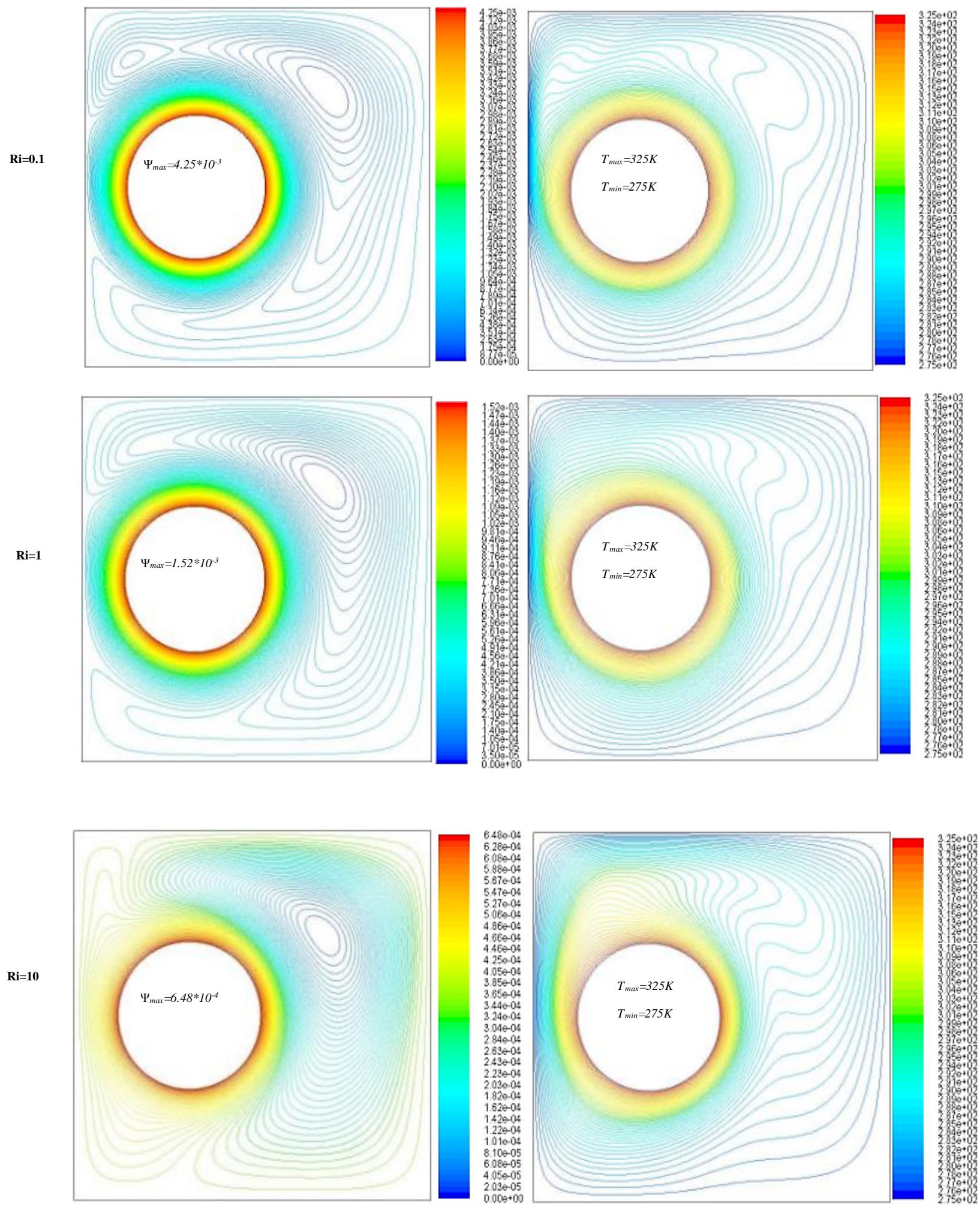


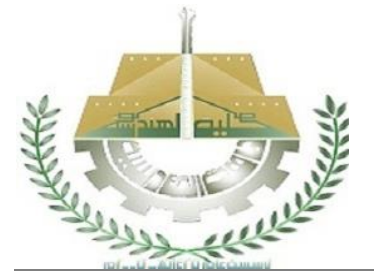

\section{AL-QADISIYAH JOURNAL FOR ENGINEERING SCIENCES}

Vol. 11, No. 1

ISSN: $1998-4456$
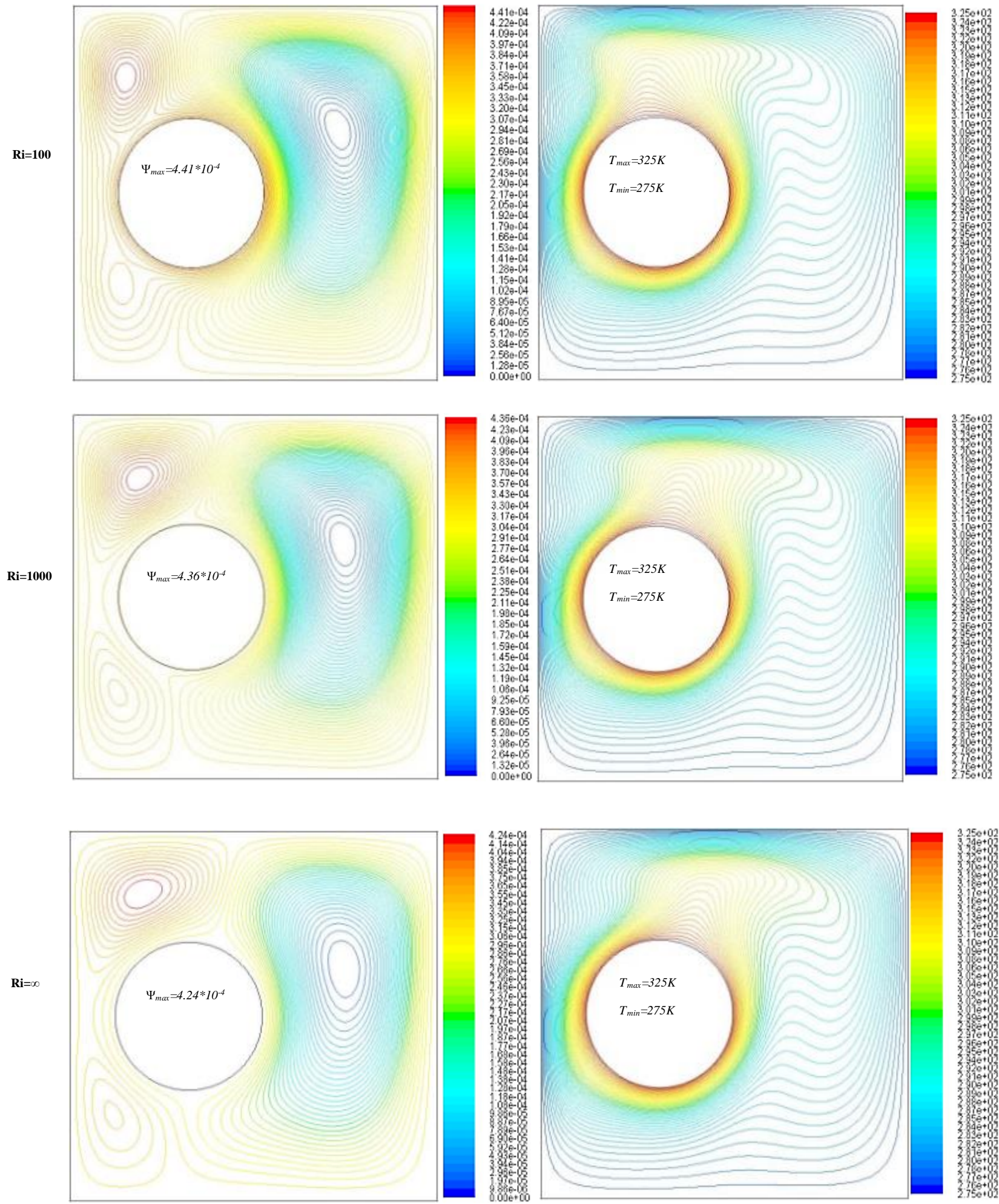

Figure 7:streamlines (on the left) and Isotherms (on the right) for different Richardson Number at Position Number Three 


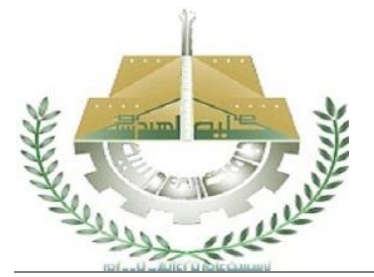

\section{AL-QADISIYAH JOURNAL FOR ENGINEERING SCIENCES}

Vol. 11, No. 1

ISSN: $1998-4456$
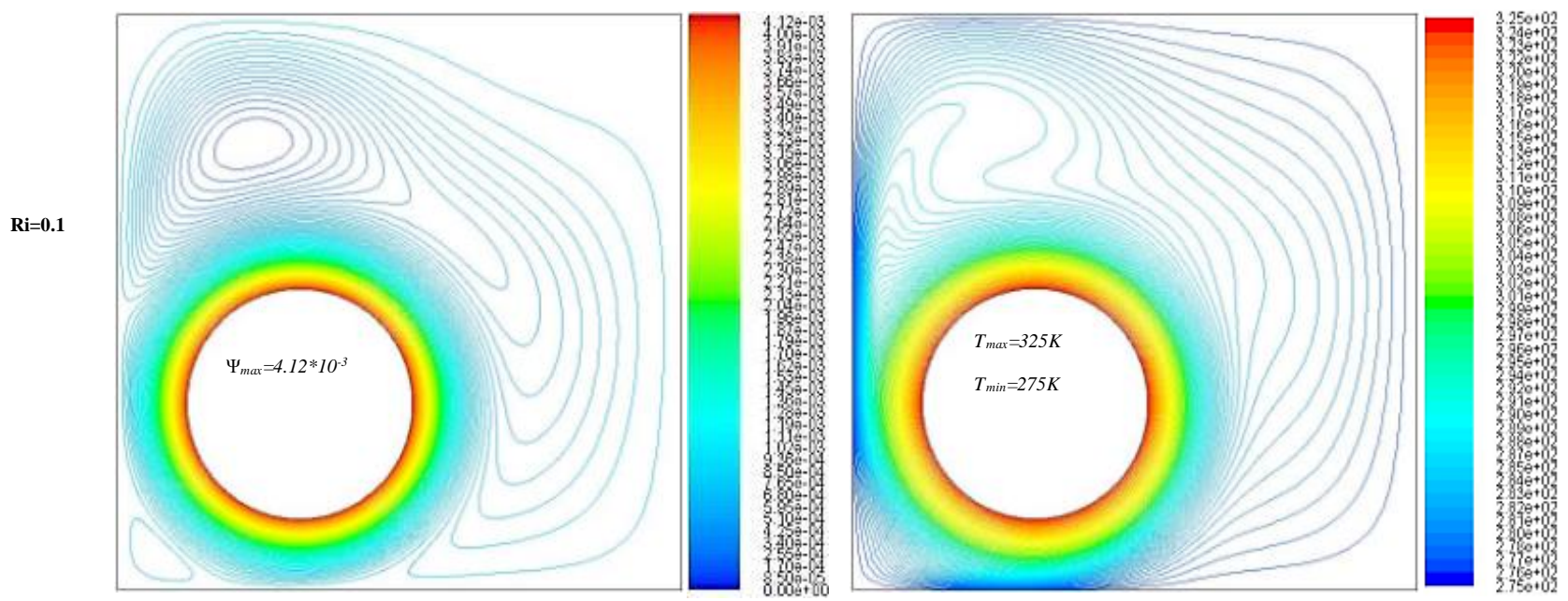

$\mathbf{R i}=\mathbf{1}$
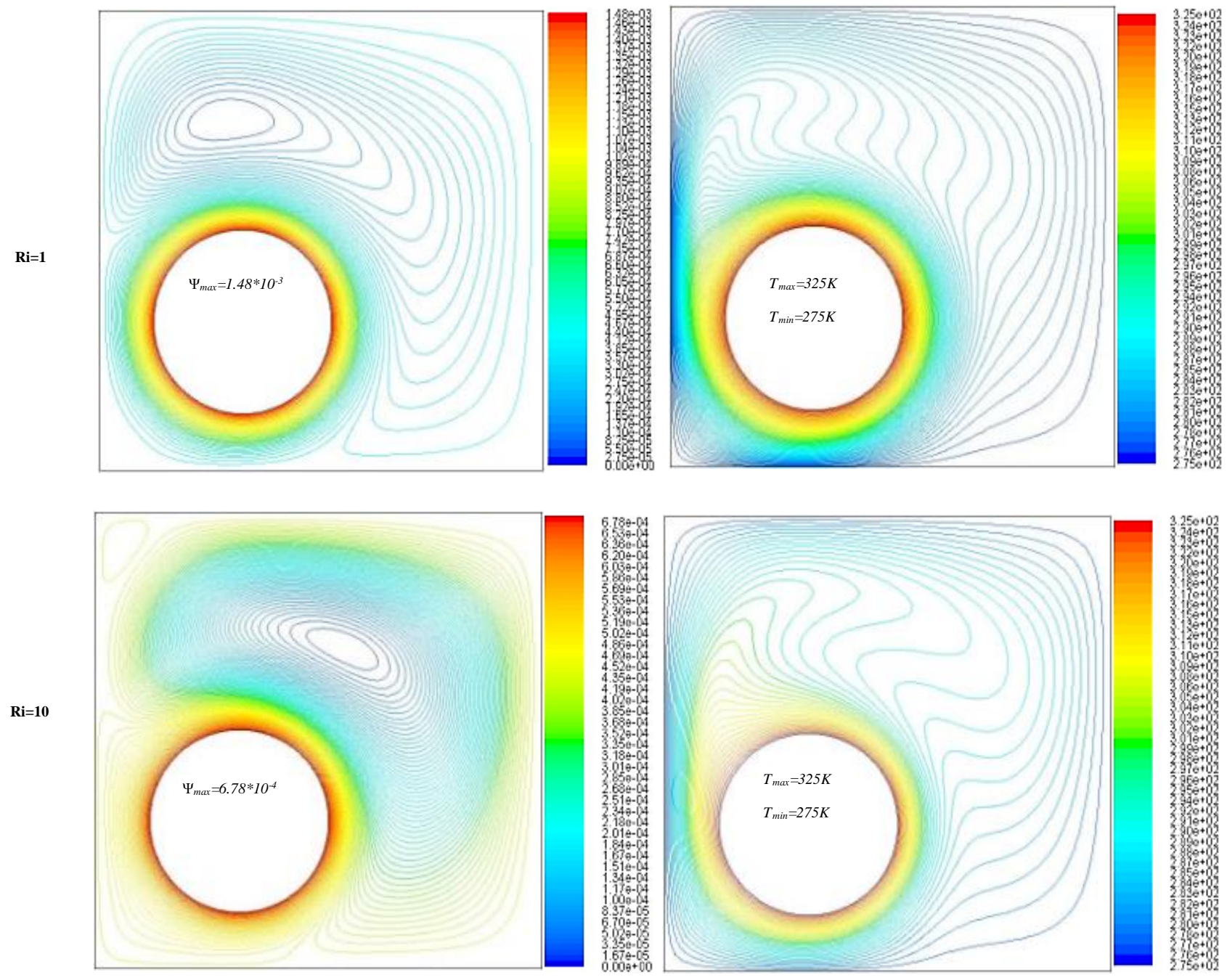


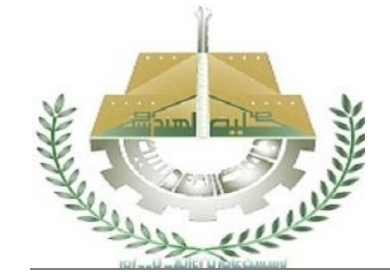

\section{AL-QADISIYAH JOURNAL FOR ENGINEERING SCIENCES}

Vol. 11, No. 1

ISSN: 1998-4456

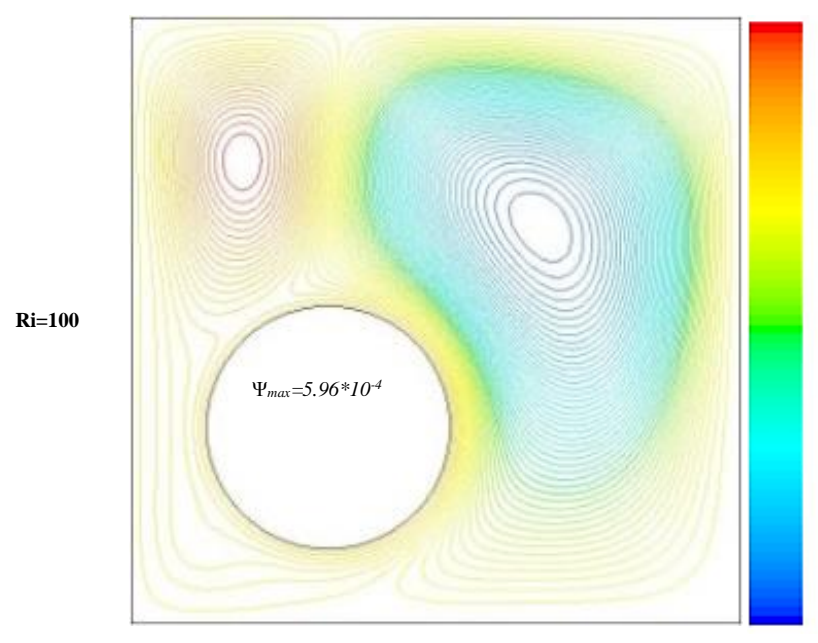

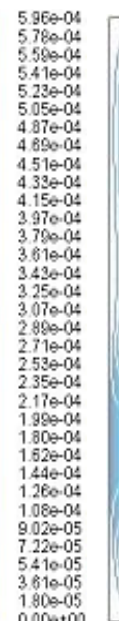
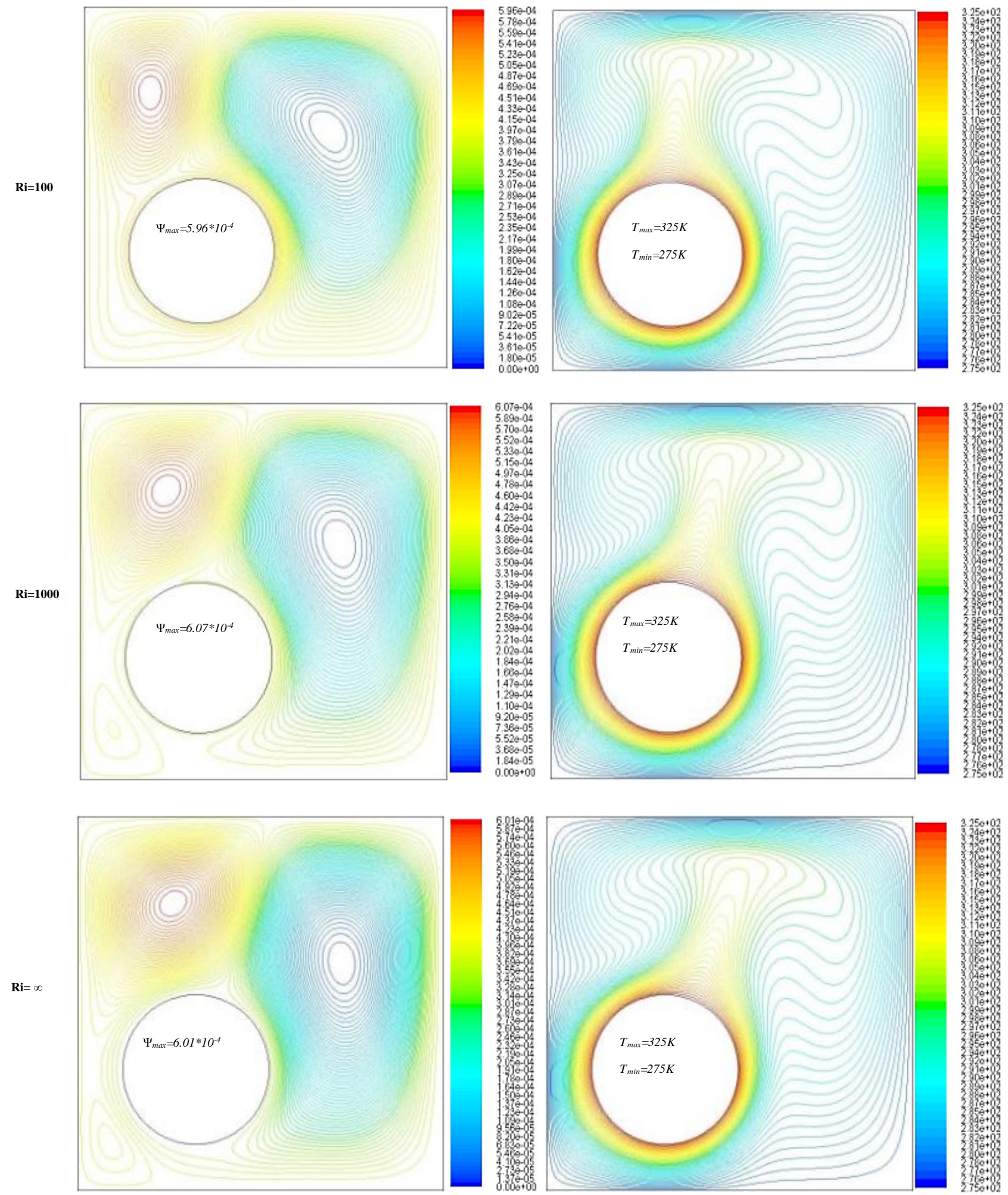

Figure 8. streamlines (on the left) and Isotherms (on the right) for different Richardson Number at Position Number Four 


\section{AL-QADISIYAH JOURNAL FOR ENGINEERING SCIENCES}

to strong the nature heat convection and gives the best combined of heat convection between forced and natural . Isotherms line behaves in same trends of streamlines where at $\mathrm{Ri}=0.1$ and $\mathrm{Ri}=1$ the effect of natural convection is started to appear at the top of cylinder and with increasing of natural heat convection due to increasing of $\mathrm{Ri}$, the isotherms line of natural convection starting dense and shifted to the right of cylinder top indicated the strong combined between the effect of forced convection and natural convection.

Fig (9) represents streamlines distribution (on the left) and Isotherms lines (on the right) for different Richardson Number at Position Number Five. From this fig, it can be noticed the position of hot rotating cylinder at lower right corner of enclave. Again at $\mathrm{Ri}=0.1$ the influence of forced heat convection is the master with limited effect to natural convection where the speed of hot cylinder is high. Gradually the influence of natural heat convection is developed and appeared at $R i=10, R i=100, R i=100$, and $R i=\infty$. As in position 4, position 5 has the mirror of position 4 but the direction of hot cylinder rotating is became in opposed to the air natural moving and as a result of that weak the combined between the natural convection and forced convection. This meaning is described fully at fig (9) stream lines distribution. Where dense of vortex on lift and above the cylinder is less in compare with same stream lines at fig (8) when the hot cylinder was in position 4 . Isotherms line reflected the same physical explanation to this phenomenon at position five. The isotherms lines indicated the starting points of initiate the natural convection effect at the lift of cylinder bottom and move with cylinder till the lift of cylinder top. In meanwhile at fig (8), the isotherms there start from right side and moved in the same direction of hot cylinder rotation.

At last but not least, fig (9) streamlines distribution (on the left) and Isotherms lines (on the right) for different Richardson Number at Position Number six. As in position 3, space for working media is available and effect of wall only on right of hot cylinder. It can be noticed at $\mathrm{Ri}=10$ the area of strong natural convection effect is just formed. Is formation is growth till the max. at $\mathrm{Ri}=\infty$ where the forced convection became weak and marginally effect the overall convection. Inside of isotherms, same scenario is repeated. Hence, at $\mathrm{Ri}=$ the natural convection influence is started and growth up side till the top of cylinder at $\mathrm{Ri}=\infty$ in opposite direction of hot cylinder rotation. The variation in air density difference is helped to move the zone of natural convection to upside.

Fig (10) gives Local Nusselt number around hot rotating cylinder for different position. From 0 to 360 degree are the positions on the surface of hot rotating cylinder and the 0 degree point at the Centre top point where the local Nusselt number is starting calculated. The local Nusselt number is analyzed and calculated for six positions of cylinder inside the enclave. For all positions of hot cylinder inside the enclave and at $\mathrm{Ri}=0.1$, the forced convection is the ruling rule on the heat convection where the variation of local Nusselt number is linear for all cylinder surface positions. Local Nusselt numbers were 21, 21.8, $19,21,21,8,19$ at hot cylinder position 1 to six respectively. It found that same Nusselt for positions 1 and four, second and fifth, and third and sixth positions respectively. The variation local Nusselt number with surface hot cylinder at $\mathrm{Ri}=1$ had same trend but marginally difference than the Nusselt number variation at $\mathrm{Ri}=0.1$. for both lines its noticeable the effect hot cylinder rotating speed on the convection. Force heat convection is major heat transfer process. Behavior tendency of local Nusselt number variation showed the starting of influence transfer from forced convection to natural convection heat transfer at cylinder position 4 clearly. Position 5 has less than clarity. At $\mathrm{Ri}=10,100,1000$, and $\infty$, the variation of local Nusselt number with position around the hot cylinder is fluctuated. 


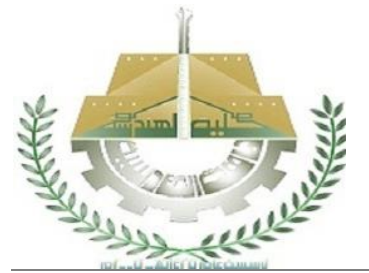
AL-QADISIYAH JOURNAL FOR ENGINEERING SCIENCES

Vol. 11 , No. 1

ISSN: $1998-4456$
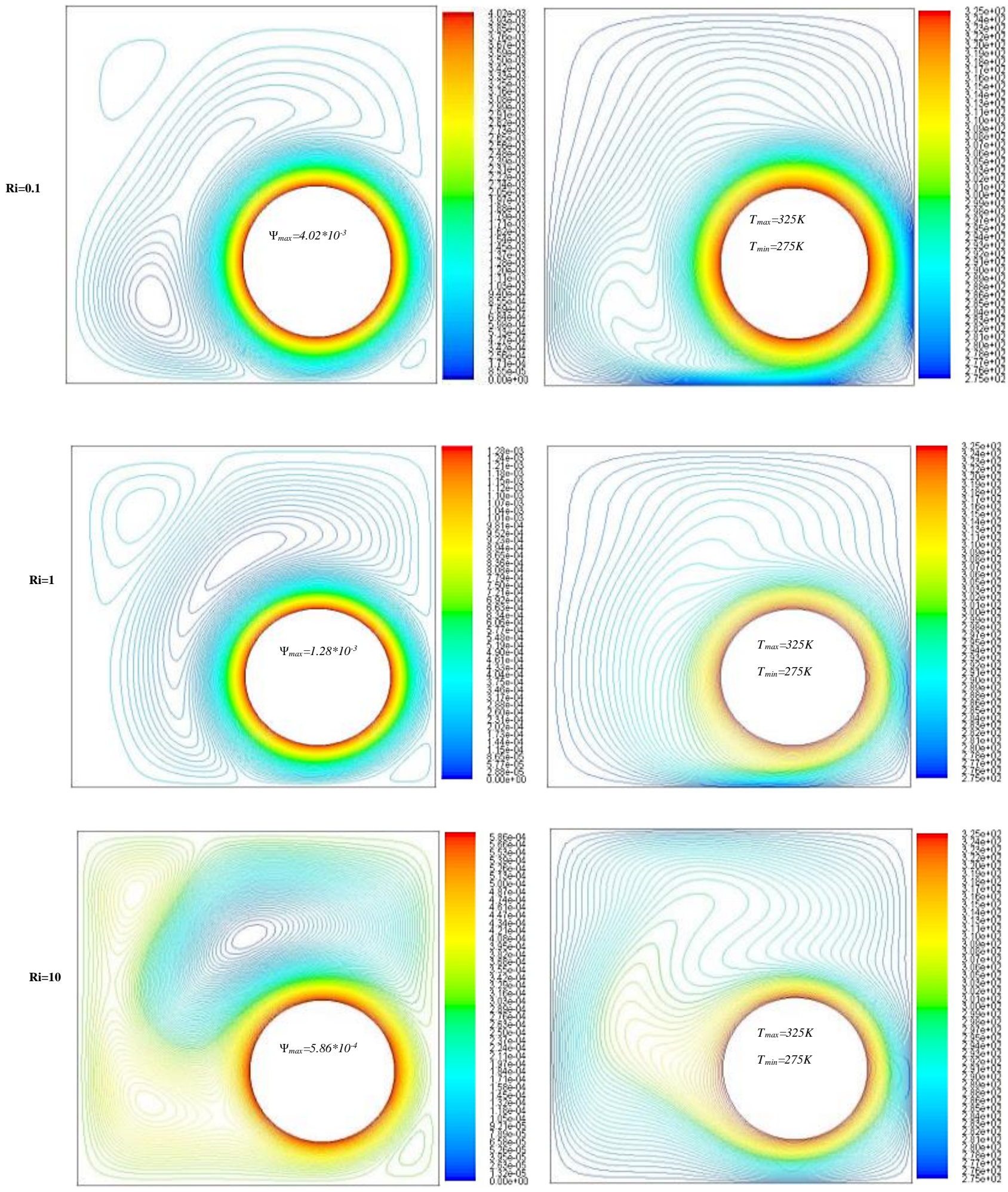


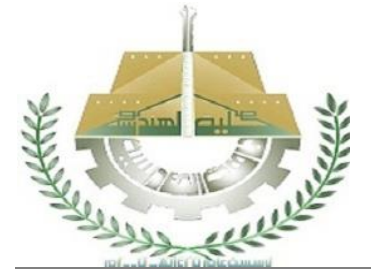

\section{AL-QADISIYAH JOURNAL FOR ENGINEERING SCIENCES}
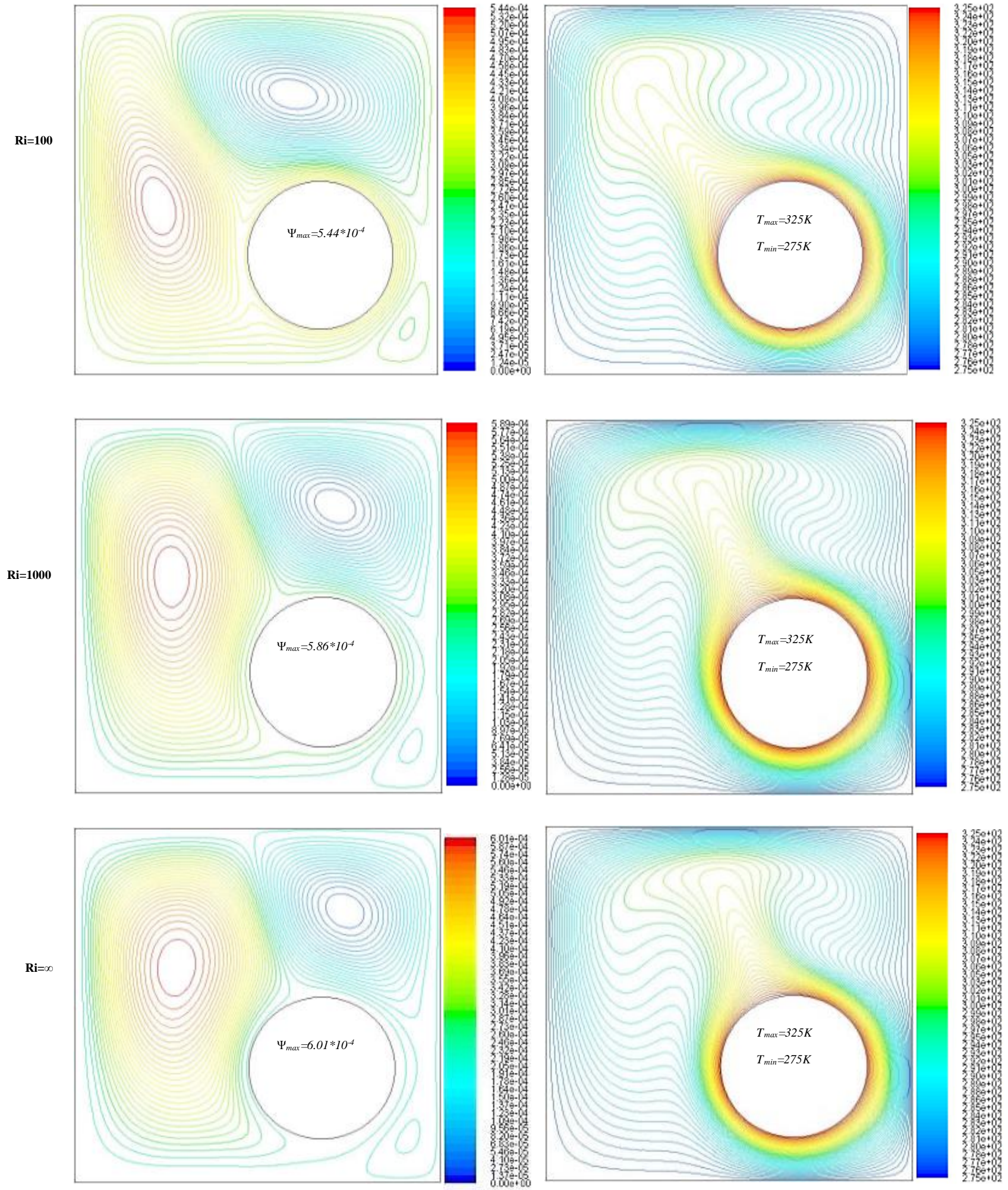

Figure 9: streamlines (on the left) and Isotherms (on the right) for different Richardson Number at Position Number Five 


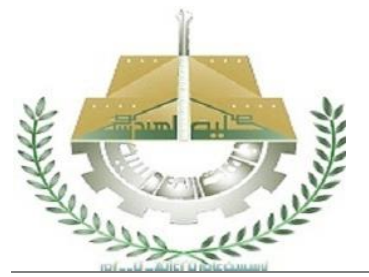
AL-QADISIYAH JOURNAL FOR ENGINEERING SCIENCES

Vol. 11, No. 1
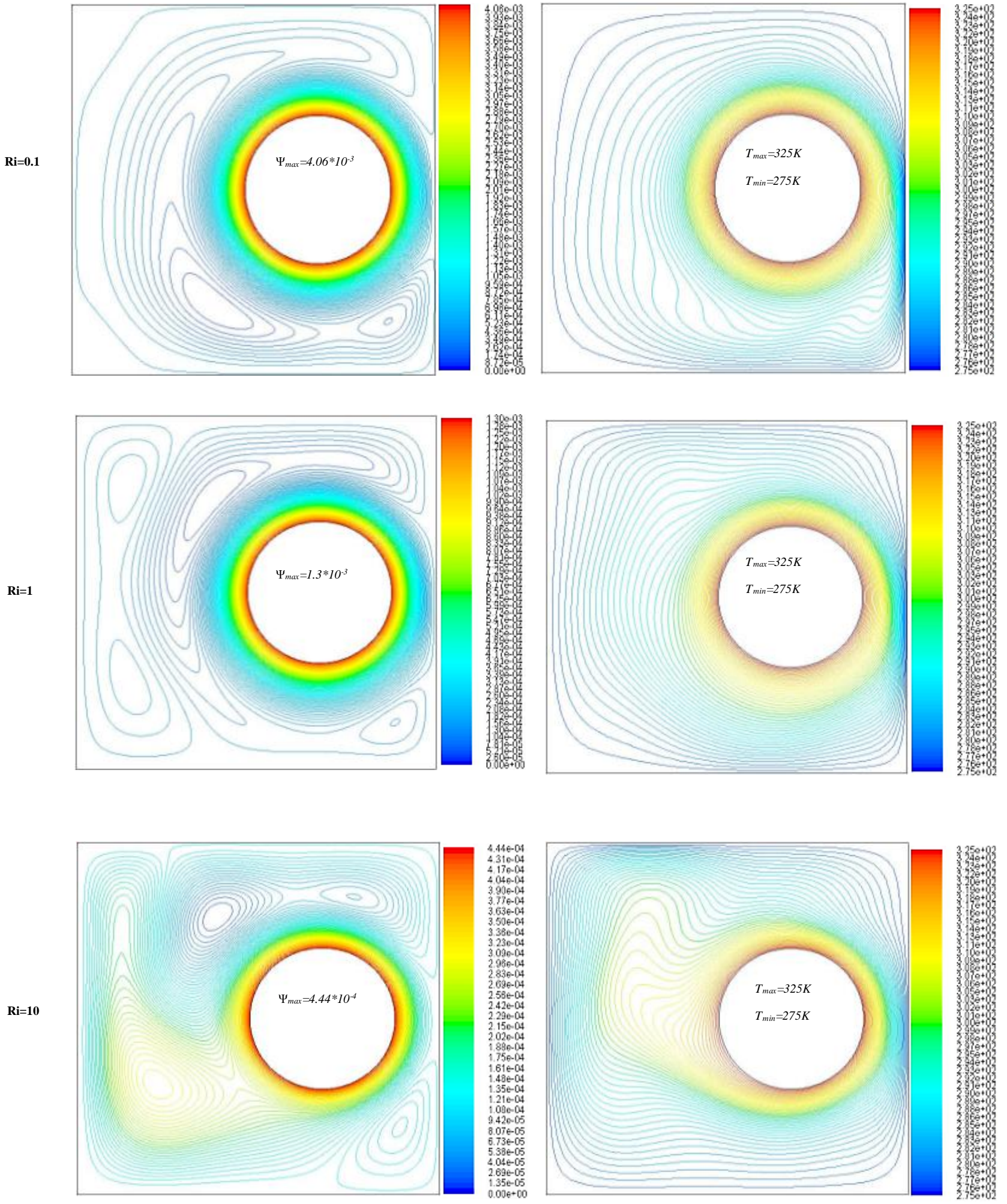


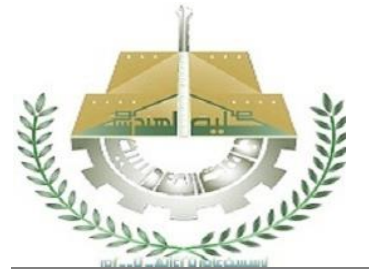

\section{AL-QADISIYAH JOURNAL FOR ENGINEERING SCIENCES}
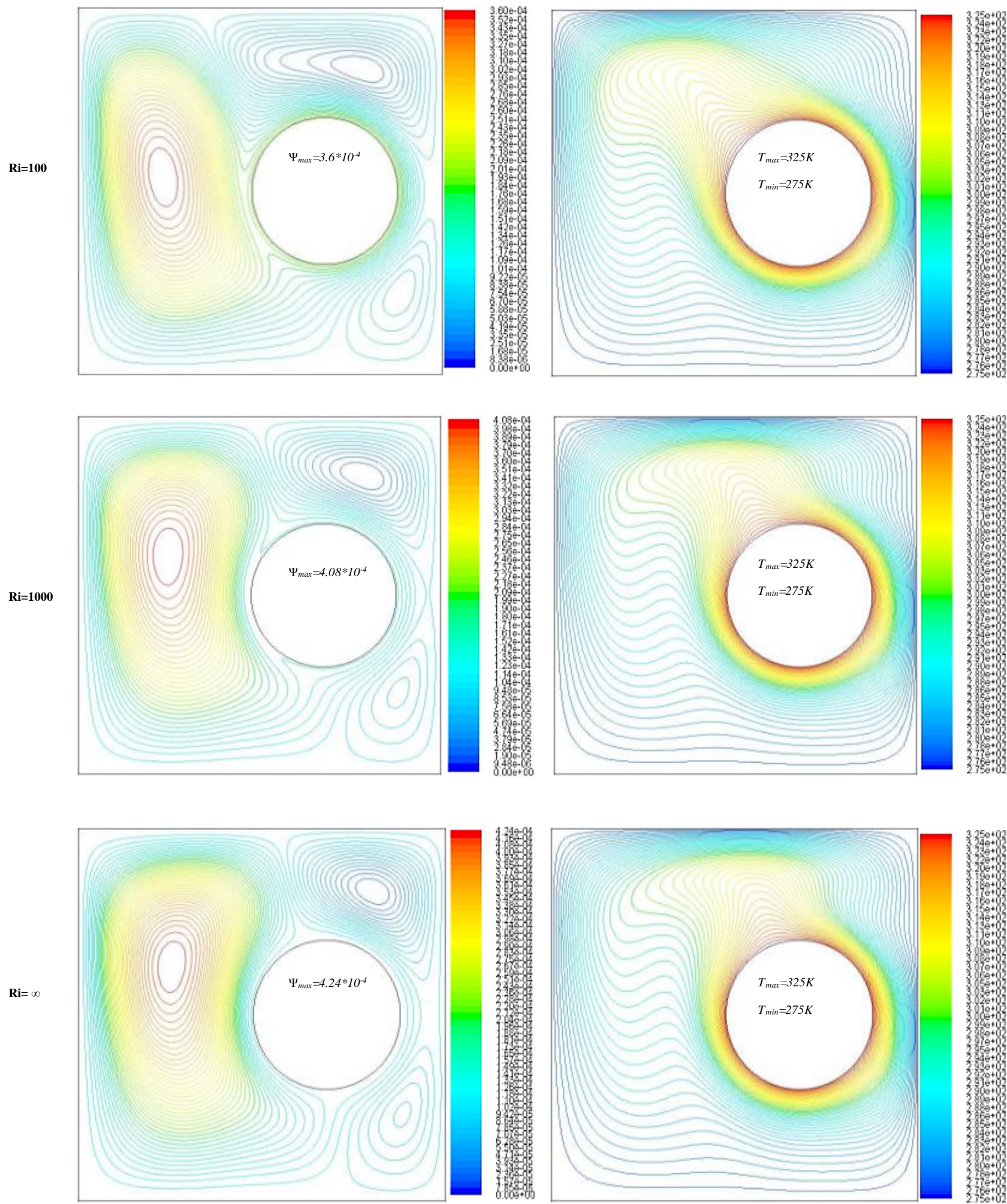

Figure 10: streamlines (on the left) and Isotherms (on the right) for different Richardson Number at Position Number Six 


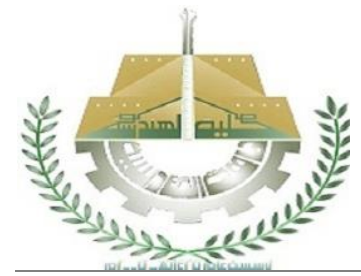

It means the effect of hot cylinder rotating speed on the heat convection is cancelled. Only major effect is declared by natural convection. nature convection effects is increasingly grown with Ri increment. At position 4 for hot cylinder, the local Nusselt number is started approximately from 28 to 30 for different Ri numbers. This point proof that position 4 is the best for best convection heat transfer. Fig (12) shows the variation of average Nusselt number with location on the hot surface. These averages is calculated by take the integral to local Nusselt number or find the accumulated area under the curve. This fig shows that position 4 is the best location to gain maximum heat transfer. In the second rank position number 5 .

At $\mathrm{Ri}=10,100,1000$, and $\infty$ the local Nusselt number is varied and has not same value at different cylinder surface degree. As shown in fig. 11 where local Nusselt number around hot Rotating Cylinder for Different Position is plotted.

Fig. 12 average Nusselt number with inverse of Richardson number for different positions are given. In this Fig. the $x$-axis represent the converse of $R i$ number (1/Ri) to cover the value of $(R i=\infty)$, therefore the meaning of the $x$-axis was converse too.

The curves show that position four gives the best combination of convection/conduction heat transfer between the hot cylinder surface and cold enclosure surface through air working media. The effect of geometric location is merely effect on the heat transfer to give the best ratio between convection and conduction.

\section{CONCLUSIONS}

The following critical points might be concluded from this numerical investigation.

1- The position of cylinder played dominant rule in the heat convection phenomenon inside closed square enclosure.

2- Stream and isothermal lines is a good indicator to the behavior and trend of heat transfer and it can be used for comparison.

3- At $\mathrm{Ri}=0.1$ and $\mathrm{Ri}=1$, the forced convection heat transfer is appear widely effect and uniform near cylinder surface while its non-uniform and less effect with Richardson number increasing.

4- Position number four is the best location for the set of cylinders to give best heat transfer process. Average Nusselt Number variation is given a good comparison to assess the best cylinder location. 


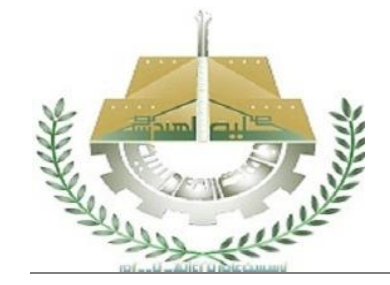

\section{AL-QADISIYAH JOURNAL FOR ENGINEERING SCIENCES}

Vol. 11 , No. 1

ISSN: $1998-4456$
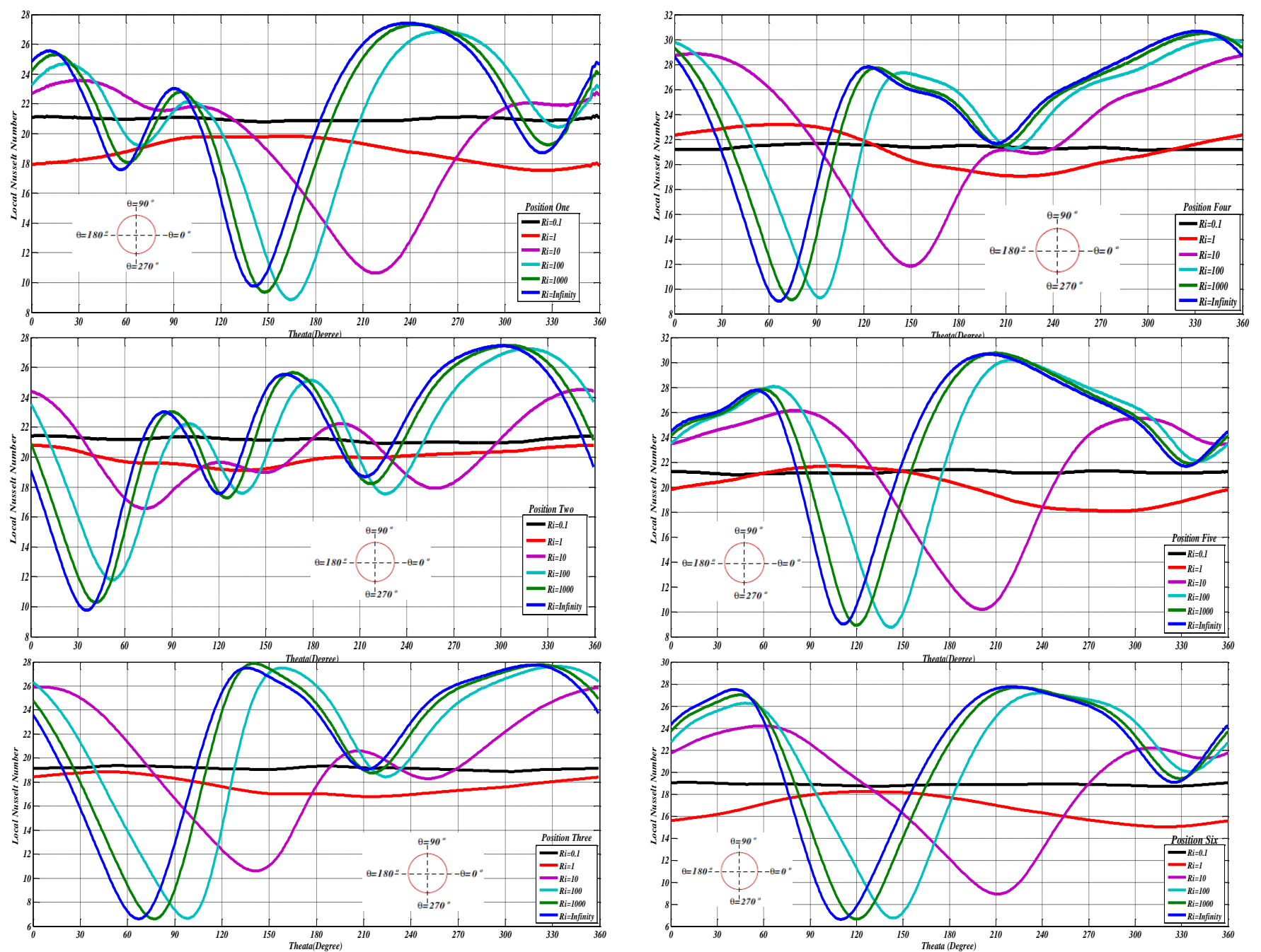

Figure 11: Local Nusselt Number around Hot Rotating Cylinder for Different Position

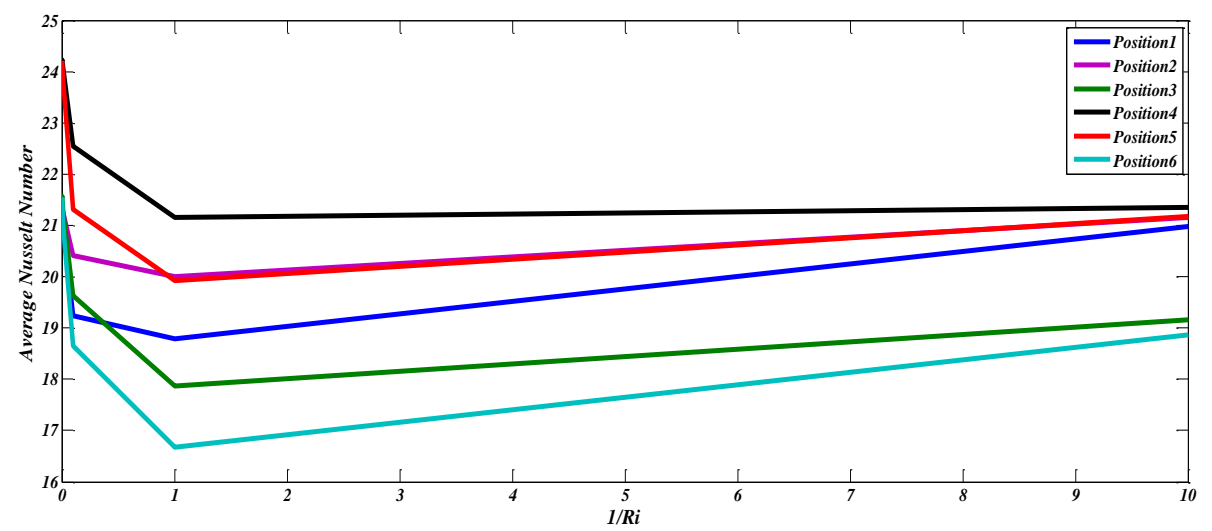

Figure 12: Average Nusselt Number with Inverse of Richardson Number for Different Position 


\section{Nomenclature}

$d \quad$ Dimensional cylinder length (m)

Gr Grashof number

\section{Greek Symbols}

$g \quad$ Gravitational acceleration $\left(\mathrm{m} / \mathrm{s}^{2}\right)$

$k \quad$ Thermal conductivity of fluid (W/m.K)

$\alpha$ Thermal diffusivity $\left(\mathrm{m}^{2} / \mathrm{s}\right)$

$H \quad$ Length of the enclosure (m)

n Normal direction

$\mathrm{Nu} \quad$ Nusselt number

$\mathrm{p} \quad$ Pressure $\left(\mathrm{N} / \mathrm{m}^{2}\right)$

$\operatorname{Pr} \quad$ Prandtl number

R Radius of circular cylinder (m)

$\beta$ Thermal expansion coefficient $(1 / \mathrm{K})$

$v \quad$ Kinematic viscosity $\left(\mathrm{m}^{2} / \mathrm{s}\right)$

$\varphi \quad$ angle of circular cylinder (degree)

$\rho \quad$ Density of the fluid $\left(\mathrm{kg} / \mathrm{m}^{3}\right)$

$\psi \quad$ Stream function

Re Reynolds number

$R i$ Richardson number

$\omega$ angular velocity ( $\mathrm{rad} / \mathrm{sec})$

$T \quad$ Temperature $(\mathrm{K})$

$\mathrm{T}_{\mathrm{c}} \quad$ Cold temperature $(\mathrm{K})$

\section{Superscripts}

$\mathrm{T}_{\mathrm{h}} \quad$ Hot temperature $(\mathrm{K})$

$u, v$ Cartesian velocity components $(\mathrm{m} / \mathrm{s})$

$X, Y$ Cartesian coordinates

\section{REFRENCES}

1. Muntasir Alam, Kamruzzaman, Faraz Ahsan, and Mohammad Nasim Hasan, " Mixed convection heat transfer inside a differentially heated square enclosure in presence of a rotating heat conducting cylinder", International Conference on Mechanical Engineering, American Insitute of physics AIP Conference Proceeding. 1754, 050035-1050035-8.

2. Mohammed Khan, Arham Amin Khan, and Mohammad Nasim Hasan, " Numerical study of mixed convection heat transfer from a rotating cylinder inside a trapezoidal enclosure", International Conference on Mechanical Engineering AIP Conf. Proc. 1754, 050036-1-050036-7.

3. Ahmed K. M. Alshara, " effect of single or multi rotatinghorizontal cylinders on the mixed convection heat transfer inside a triangular enclosure", Al-Qadisiya Journal For Engineering Sciences Vol. 5 No. 1 Year 2012.

4. Chuan-Chieh Liao, Chao-An Lin," Mixed convection of a heated rotating cylinder in a square enclosure", Int. Journal of Heat and Mass Transfer 72 (2014) pp 9-22.

5. Fatih Selimefendigil, Hakan F. Oztop " Numerical study of MHD mixed convection in a nanofluid filled lid driven square enclosure with a rotating cylinder", Int. Journal of Heat and Mass Transfer 78 (2014) pp741-754. 
6. Salam Hadi Hussain, Ahmed Kadhim Hussein," Mixed convection heat transfer in a differentially heated square enclosure with a conductive rotating circular cylinder at different vertical locations", Int. Communications in Heat and Mass Transfer 38 (2011) pp. 263-274.

7. Fatih Selimefendigil a, *, Hakan F. O“ztop," MHD mixed convection of nano fluid filled partially heated triangular enclosure with a rotating adiabatic cylinder", Journal of the Taiwan Institute of Chemical Engineers 45 (2014) pp 2150-2162.

8. Chuan-Chieh Liao, Chao-An Lin, "Mixed convection of a heated rotating cylinder in a square enclosure", International Journal of Heat and Mass Transfer • May 2014.

9. Paulo Mohallem Guimarães, Doacir Vilar de Assis Junior, Genésio José Menon, " laminar natural convection in a square enclosure with in internal rotating heated cylinder", Proceedings of the 10o Brazilian Congress of Thermal Sciences and Engineering - ENCIT- 2004. Braz. Soc. of Mechanical Sciences and Engineering -- ABCM, Rio de Janeiro, Brazil, Nov. 29 -- Dec. 03, 2004.

10. Aydin Misirlioglu, "The effect of rotating cylinder on the heat transfer in a square cavity filled with porous medium", International Journal of Engineering Science 44 (2006) 1173-1187.

11. V.A.F. Costa, A.M. Raimundo," Steady mixed convection in a differentially heated square enclosure with an active rotating circular cylinder", International Journal of Heat and Mass Transfer 53 (2010) 1208-1219.

12. Fariborz Karimi, Hongtao Xu, Zhiyun Wang, Mo Yang, Yuwen Zhang, " Numerical simulation of transient forced convection in a square enclosure containing two heated circular cylinders", 233_Talkhoncheh_IJNMHFF_2016.

13. Yu-fei WANG, Xu XU, Tian TIAN, Li-wu FAN, Wen-long WANG, Zi-tao YU," Laminar mixed convection heat transfer of SiC-EG nanofluids in a triangular enclosure with a rotating inner cylinder: simulations based on the measured thermal conductivity and viscosity", J Zhejiang Univ-Sci A (Appl Phys \& Eng) 2015 16(6):478-490.

14. Chuan-Chieh Liao, Chao-An Lin "Mixed convection of heated rotating cylinder in a square enclosure" International Journal of Heat and Mass Transfer 72 (2014) 9-22.

15. Ansys Fluent Theory Guide, Release 16.0. 
